\title{
El control del tabaco, estrategia esencial para reducir las enfermedades crónicas no transmisibles
}

\author{
Luz Myriam Reynales-Shigematsu, D en C. ${ }^{(1)}$
}

\section{Reynales-Shigematsu LM. El control del tabaco, estrategia esencial para reducir las enfermedades crónicas no transmisibles. Salud Publica Mex 20I 2:54:323-33I.}

\section{Resumen}

Cerca de dos terceras partes del total de muertes a nivel global son causadas por las enfermedades crónicas no transmisibles. Se han recomendando cinco intervenciones prioritarias para disminuir esta tendencia: I. Control del tabaco (la más urgente e inmediata), 2. Reducción del consumo de sal, 3. Mejoría en la dieta y actividad física, 4. Reducción del consumo peligroso de alcohol y 5 . Acceso a los medicamentos esenciales y la tecnología. En relación con los padecimientos derivados del consumo del tabaco, la OMS reconoce el conflicto fundamental de intereses entre las tabacaleras y la salud pública y sugiere la implementación del Convenio Marco para el Control del Tabaco de la OMS y las estrategias MPOWER, ya que su completa implementación podría evitar cerca de 5.5 millones de muertes en los siguientes 10 años. Todas estas recomendaciones son viables y factibles de implementar si se consideran la voluntad política de los gobiernos, la infraestructura disponible, la capacidad técnica existente, la participación coordinada de todos los sectores y grupos de interés, la sociedad civil organizada y la colectividad en su conjunto.

Palabras claves: tabaco; enfermedad crónica; prevención y control; objetivos de desarrollo del milenio
Reynales-Shigematsu LM.

Tobacco control, a strategy to reduce

non-communicable diseases.

Salud Publica Mex 20I 2:54:323-33I.

\begin{abstract}
Nearly two-thirds of all deaths globally are caused by noncommunicable diseases (cardiovascular diseases, cancer, respiratory diseases and diabetes). The UN General Assembly approved Political Declaration of the High-Level Meeting on the Prevention and Control of non communicable diseases and recommending five priority interventions: I. Tobacco control (the most urgent and immediate), 2. Salt reduction, 3. Improved diet and physical activity, 4 Reduction of hazardous alcohol intake, 5. Access to essential drugs and technologies. The Assembly recognizes the fundamental conflict of interest between tobacco industry and public health and recommends the implementation of WHO Framework Convention on Tobacco Control (FCTC) and MPOWER strategies. The full implementation of FCTC could prevent 5.5 Million of death in the next 10 years in low and middle income countries. All these recommendations are feasible to implement considering the willingness of Governments, the infrastructure available, the capacity building existing and the participation of all sectors, including civil society and the community as a whole.
\end{abstract}

Key words: tobacco; chronic disease; prevention \& control; millennium development goals

(I) Departamento de Investigación sobre Tabaco, Centro de Investigación en Salud Poblacional, Instituto Nacional de Salud Pública. Cuernavaca, Morelos, México.

Fecha de recibido: 6 de marzo de 2012 - Fecha de aprobado: 20 de marzo de 2012

Autor de correspondencia: Luz Myriam Reynales Shigematsu. Departamento de Investigación sobre Tabaco, Centro de Investigación en Salud Poblacional, Instituto Nacional de Salud Pública. Av. Universidad 655, col. Santa María Ahuacatitlán. 62100 Cuernavaca, Morelos, México.

Correo electrónico: Ireynales@insp.mx 


\section{Consumo y exposición a humo de tabaco, factor de riesgo causal de las enfermedades crónicas no transmisibles}

En 2008, cerca de dos terceras partes del total de muertes en el ámbito global, 36 millones, fueron causadas por las enfermedades crónicas no transmisibles (ECNT), principalmente enfermedades cardiovasculares (48\%), cáncer $(21 \%)$, enfermedades respiratorias crónicas (12\%) y diabetes (3\%). El 25\% de las muertes ocurrieron en personas menores de 60 años, reduciendo significativamente la productividad laboral. ${ }^{1}$ Proyecciones realizadas para el 2020 estiman que las ECNT causarán 44 millones de muertes al año; en los siguientes 20 años, las ECNT costarán más de 30 trillones de dólares, 48\% del producto interno bruto (PIB) global en 2010, lo que trae como consecuencia millones de personas por debajo de la línea de pobreza. ${ }^{2}$

Los principales factores de riesgo comunes que causan las ECNT son el consumo de tabaco, una dieta malsana, inactividad física y el abuso del alcohol. Un poco más de 1000 millones de personas fuman tabaco en todo el mundo; su consumo causa 1 de cada 6 muertes por ECNT y es factor de riesgo de seis de las ocho principales causas de mortalidad en el mundo, ${ }^{3}$ lo que ocasiona de manera prematura cerca de 6 millones de muertes anuales. ${ }^{4}$ El consumo de tabaco se mantiene hoy día como la principal causa de muerte prevenible a nivel mundial.

Adicionalmente, la Organización Mundial de la Salud (OMS) estima que alrededor de 700 millones de niños, casi la mitad de la población infantil mundial, respiran aire contaminado por humo de tabaco, y es la exposición en el hogar la de mayor importancia. ${ }^{5}$ La exposición al humo del tabaco contribuye en gran medida a la carga mundial de la enfermedad atribuible al tabaquismo. ${ }^{6}$

De continuar el patrón de consumo actual, el número de muertes anuales atribuibles al tabaquismo aumentará a 10 millones anuales en 2030; se estima que el tabaco podría matar a cerca de 1000 millones de fumadores durante el siglo XXI y el 80\% de estas muertes prematuras ocurrirán en los países de ingresos bajos y medios. ${ }^{4,-9}$ Cerca de la mitad de los fumadores morirá entre los 30 y 69 años de edad y perderán por lo menos una década de vida productiva. ${ }^{8}$

\section{La epidemia del tabaquismo converge con las epidemias de origen infeccioso}

En una visión integral de la situación epidemiológica mundial, el consumo de tabaco y la exposición a su humo no sólo están asociados con la epidemia causada por las ECNT; también converge y en especial en los países de bajos y medianos ingresos, con epidemias infecciosas como la tuberculosis (TB), el virus de inmunodeficiencia humana (VIH) y la influenza. ${ }^{10}$ Estudios de metaanálisis refieren que el consumo de tabaco se encuentra asociado con la infección latente por TB, la progresión hacia la enfermedad activa, la muerte y la recaída de la enfermedad. ${ }^{11}$ De igual manera, es un factor de riesgo independiente para los pacientes fumadores que han contraído una infección por VIH, los cuales tienen mayor carga viral y una tasa de progresión de la enfermedad mayor si se compara con los no fumadores. ${ }^{11}$

El consumo de tabaco se asocia con infecciones respiratorias y neumonías, principalmente causadas por Streptococo, Legionella, Mycoplasma y Haemophilus influenzae. Se ha documentado que los fumadores tienen mayor riesgo individual de infección por influenza, pues presentan los cuadros más severos y complicados. Adicionalmente, la eficacia de la vacunación es reducida en este grupo poblacional. ${ }^{12}$ Aunque hay muy pocos datos de la asociación del consumo de tabaco y la reciente pandemia por H1N1; dado que los estudios no reportaron el status del fumador, es importante tenerlo presente, pues coexisten a nivel global. ${ }^{10}$

\section{La transición epidemiológica, demográfica y social de la epidemia de tabaquismo}

La relación entre el tabaquismo, los daños a la salud y su impacto en la morbimortalidad están ampliamente documentados desde el punto de vista científico. ${ }^{13,14}$ De manera paradójica, la magnitud de sus efectos no son percibidos por la población general ni por los fumadores, lo que se debe en gran medida a la falta de comprensión del comportamiento y la evolución del factor de riesgo, pero de manera particular, en el tiempo que transcurre entre la instauración de la epidemia y el incremento masivo de las tasas de mortalidad, lo cual, en el caso del tabaquismo, puede tomar de tres a cuatro décadas en llegar a ser completamente evidente. ${ }^{15}$ De igual manera, los fumadores no son conscientes de la rápida disminución del riesgo que ocurre inmediatamente después de abandonar el consumo de tabaco y que se observa de manera evidente en la enfermedad coronaria. ${ }^{16}$

En menos de una década, la mortalidad atribuible al tabaco se ha incrementado en casi dos millones de muertes anuales y la carga de la mortalidad ha pasado de 50 a más de $80 \%$ en los países de bajos y medianos ingresos..$^{7-9}$ La pandemia global del tabaco se mueve hacia los países en desarrollo y se focaliza en la población más vulnerable (mujeres, jóvenes y grupos con 
menores ingresos económicos). En los próximos años, las tasas de mortalidad atribuibles a tabaco caerán en los países desarrollados pero continuarán incrementándose a nivel global a menos que los fumadores de los países en desarrollo dejen de fumar antes de los 35 años..$^{7-9}$ Por otro lado, las proyecciones prevén que aun cuando las prevalencias de consumo tendieran a disminuir, el número absoluto de fumadores aumentaría dado el crecimiento poblacional. ${ }^{17}$ Esta transición epidemiológica y demográfica que varía a través del tiempo y entre las regiones del mundo, también se encuentra asociada con las características socioeconómicas de las poblaciones. Al hacer un paralelo entre el modelo de las cuatro etapas de la epidemia de tabaquismo en los países desarrollados propuesto por López $\mathrm{AD},{ }^{15}$ varios autores proponen una transición socioeconómica de la epidemia: en las etapas iniciales se disemina entre los grupos de mayores ingresos, posiblemente por estar más abiertos a la innovación; durante las etapas intermedias se difunde al resto de la población, para finalmente declinar entre los grupos socioeconómicos altos como consecuencia de un mayor grado de conciencia de la salud. ${ }^{18}$ Aunque este modelo no necesariamente aplica a todos los países, es importante visualizar la perspectiva.

El consumo de tabaco causa un daño inmediato en los fumadores y sus familias; poco a poco, cada día, los debilita y con el tiempo los empobrece. El dinero que gastan en tabaco deja de invertirse en alimentos y otras necesidades básicas como educación y salud. ${ }^{18}$ Las enfermedades y la muerte prematura de los fumadores reducen en principio el ingreso familiar, pero sus consecuencias podrían ser devastadoras si esto ocurriera en una familia que está muy cerca o por debajo de la línea de pobreza, pues tendrían que decidir entre buscar el sustento alimenticio de la familia o atender su salud, lo que los hace vulnerables y, en consecuencia, se crea una gran inequidad social. ${ }^{18,19}$

\section{La globalización de la epidemia}

La globalización de la epidemia de tabaquismo obedece a una interacción mixta y compleja de factores, con efectos transfronterizos que incluyen la liberalización del comercio y la inversión extranjera directa. ${ }^{20}$ Otros factores como el carácter transnacional y monopólico de la industria del tabaco (IT), sus nuevas estrategias de mercadotecnia mundial dirigidas a captar el mercado adolescente, el cabildeo que realiza entre los grupos de influencia, ${ }^{21}$ además del movimiento internacional del contrabando y la falsificación de cigarrillos, han contribuido en el incremento explosivo del consumo de tabaco a nivel global.

\section{La epidemia en México, la carga de la enfermedad y su impacto económico y social}

México no escapa a esta epidemia globalizada. La Encuesta Global de Tabaquismo en Adultos (GATS) realizada en México en el año $2009^{22}$ refiere que 15.9\% (10.9 millones) de los adultos en México son fumadores actuales, $24.8 \%$ de los hombres (8.1 millones) y $7.8 \%$ (2.8\% millones) de las mujeres. El $48 \%$ de los fumadores actuales son fumadores diarios. El 99\% de los hombres y $96 \%$ de mujeres fuman cigarrillos manufacturados. Entre los fumadores diarios de cigarrillos, en promedio, los hombres fuman 9.7 cigarrillos por día y las mujeres 8.4. La edad de inicio de fumar entre los fumadores diarios de 18 a 43 años fue de 16.3 años para los hombres y 16.8 años para las mujeres.

La Encuesta de Tabaquismo en Jóvenes (ETJ) realizada en 32 ciudades capitales de México durante 2005, 2006 y 2008, ${ }^{23}$ en estudiantes entre 13 y 15 años de edad estima una prevalencia de consumo de tabaco que oscila entre 10.5 y $27.8 \%$. Los adolescentes inician el consumo de tabaco en edades cada vez más tempranas, generalmente antes de cumplir los 15 años. La ETJ encontró que entre 8 y 15\% de los estudiantes ha probado el cigarrillo antes de los 13 años de edad y $7.6 \%$ de los estudiantes fuman más de 6 cigarrillos al día, lo cual es signo de adicción a la nicotina en este grupo de edad.

De acuerdo con la Encuesta Nacional de Adicciones (ENA 2008), ${ }^{24}$ en el ámbito nacional cerca de 11 millones (23.3\%) de mexicanos que nunca han fumado, se encuentran expuestos al humo de tabaco ambiental (HTA); $25.5 \%$ son hombres ( 4.7 millones) y $22 \%$ mujeres (6.5 millones). La ETJ de 2005, 2006 y 2008, encontró que los estudiantes entre 13 y 15 años de edad estaban expuestos al HTA tanto en los lugares públicos como en sus hogares. En 2005, Barrientos y colaboradores ${ }^{25}$ realizaron un estudio para cuantificar los niveles de exposición al humo de tabaco en hogares mexicanos y encontró una correlación directa entre el número de fumadores en el hogar y las concentraciones de nicotina en el cabello de los no fumadores, observando los más altos grados de exposición entre los niños.

Otro grupo poblacional de especial interés por su rol en el control del tabaco son los estudiantes de profesiones relacionadas con la salud. La Encuesta de Tabaquismo en Estudiantes de la Salud, ${ }^{26}$ aplicada en 2007 a estudiantes de tercer año de medicina y odontología de universidades públicas y con representatividad nacional, encontró una prevalencia de fumadores de $33.3 \%$ en estudiantes de medicina y de $43.6 \%$ en estudiantes de odontología, sin que existieran diferencias 
estadísticamente significativas entre hombres y mujeres. Más de $80 \%$ de los estudiantes que respondieron a esta encuesta tenían entre 19 y 24 años, que corresponde al grupo de edad donde se presenta la mayor prevalencia de fumadores en el país.

La Encuesta Nacional de Salud y Nutrición (ENSANUT 2006), ${ }^{27}$ la Encuesta Nacional de Adicciones (ENA) y el Sistema Global de Vigilancia Epidemiológica del Tabaco (GTSS) confirman que la epidemia de tabaquismo en México continúa siendo un grave problema de salud pública, con una tendencia al incremento entre los adolescentes, adultos jóvenes (18-25 años) y las mujeres, tanto en las áreas urbanas como rurales. A pesar de que la prevalencia de consumo está dada principalmente por fumadores ocasionales y fumadores diarios que consumen en promedio pocos cigarros por día, es preciso recordar que no existen niveles seguros de consumo de tabaco. ${ }^{28}$ Por lo tanto, la mejor opción es que los fumadores dejen completamente de fumar y que los adolescentes nunca inicien el consumo.

Valdés-Salgado ${ }^{29}$ estimó que en el año 2000 ocurrieron cerca de 25383 muertes prematuras atribuibles al consumo de tabaco, de las cuales 5165 fueron por neoplasias, 9057 por enfermedades respiratorias, 5345 por enfermedades cardiovasculares y 5816 por otras causas médicas relacionadas con el consumo de tabaco. Las enfermedades relacionadas con su consumo directo e indirecto, como el infarto agudo del miocardio, las enfermedades cerebrovasculares, las respiratorias crónicas y el cáncer de pulmón continúan en el México de hoy dentro de las diez primeras causas de mortalidad. ${ }^{30}$

Sáenz de Miera B y colaboradores, ${ }^{31}$ en su estudio "Las repercusiones del consumo de tabaco en la distribución del gasto de los hogares mexicanos", encontraron que en términos relativos los hogares fumadores más pobres de México destinan una porción mayor de su gasto total a cigarrillos comparado con los hogares más ricos. Un hogar fumador pobre gasta en promedio 211 pesos $^{*}$ en tabaco por mes, lo cual representa $5.5 \%$ de su gasto total y la octava parte de su gasto en alimentos. Los hogares fumadores más ricos gastan en promedio 360 pesos por mes en tabaco, lo cual representa 3.1\% de su gasto total. ${ }^{32} \mathrm{Al}$ igual que en otros estudios internacionales ${ }^{18}$ se encontró que los hogares fumadores en México gastan menos en alimentación y educación comparado con los hogares no fumadores; este hallazgo fue independiente del nivel de ingreso. ${ }^{31}$

\footnotetext{
* Cantidades en pesos corresponden a pesos mexicanos de 2010.
}

Los hogares fumadores pobres gastaron al menos 485 millones de pesos en productos de tabaco en 2010, lo cual representa $13 \%$ del presupuesto para el Programa de Apoyo Alimentario de ese año. En total, los hogares fumadores gastaron al menos 6372 millones de pesos en productos de tabaco en $2010 .{ }^{31}$

Estudios recientes del impacto económico del consumo de tabaco en México estima que los costos anuales de atención médica atribuibles al consumo activo de tabaco en México de las cuatro principales enfermedades (infarto agudo de miocardio, enfermedad cerebrovascular, enfermedad pulmonar obstructiva crónica y cáncer de pulmón) oscila entre 23000 millones de pesos mexicanos, en un escenario conservador, y 43000 millones considerando un escenario alto. El infarto agudo al miocardio y el cáncer de pulmón fueron las enfermedades más costosas tanto en el escenario conservador como en el alto. ${ }^{33}$

\section{La respuesta del mundo globalizado}

En septiembre de 2011, la Asamblea General de las Naciones Unidas (UN) aprobó la Declaración Política de la Reunión de Alto Nivel de la Asamblea General sobre la Prevención y el Control de las ECNT ${ }^{34,35}$ en todo el mundo, centrándose particularmente en los problemas de desarrollo y en sus repercusiones sociales y económicas, especialmente para los países en desarrollo. En esta asamblea hay un reconocimiento claro de que la carga de enfermedad ocasionada por las ECNT son un obstáculo para el desarrollo en el siglo XXI, socavan el desarrollo social y económico, aumentan las desigualdades entre países y poblaciones (inequidad) y pueden tener consecuencias directas para el logro de los objetivos de desarrollo convenidos internacionalmente, incluidos los Objetivos de Desarrollo del Milenio (ODM) ${ }^{36}$

La Asamblea reconoce el conflicto fundamental de intereses entre la industria del tabaco y la salud pública y observa con aprecio el Convenio Marco de la OMS para el Control del Tabaco (CMCT-OMS). ${ }^{18}$ Reafirma todas las resoluciones y decisiones pertinentes aprobadas por la Asamblea Mundial de la Salud en relación con la prevención y el control de las ECNT, subrayando la importancia de que los Estados Miembros sigan haciendo frente a los factores de riesgo comunes a través de cinco intervenciones prioritarias que consideran los efectos en la salud, la costoefectividad, el bajo costo de implementación y la factibilidad financiera y política:

1. Control del tabaco (la prioridad más urgente e inmediata)

2. Reducción del consumo de sal

3. Mejoría en la dieta y actividad física 
4. Reducción del consumo peligroso de alcohol

5. Acceso a los medicamentos esenciales y la tecnología

El CMCT-OMS demuestra que hay voluntad política mundial para reforzar el control del tabaco y salvar vidas humanas. Este tratado mundial, jurídicamente vinculante, sienta las bases para que los países apliquen y gestionen programas de control para hacer frente a la epidemia creciente de consumo de tabaco. En septiembre de 2011, el CMCT reunía 174 Partes, 87\% de la población mundial, lo cual hace de él uno de los tratados que más rápidamente ha suscitado adhesiones en la historia de las Naciones Unidas. ${ }^{37}$ Para ayudar a los países al cumplimiento de las obligaciones establecidas en el CMCT, la OMS estableció en 2008 el plan de medidas MPOWER, ${ }^{3}$ el cual es parte integral del Plan de Acción de la OMS para la Prevención y el Control del Tabaco, e incluye las seis estrategias más costoefectivas que deben implementar los países miembros para abatir la epidemia del tabaquismo a nivel global:

Monitor: Monitorear el consumo de tabaco y las políticas de prevención

Protect: Proteger a las personas de la exposición al humo de tabaco ajeno

Offer: Ofrecer ayuda para dejar el consumo de tabaco

Warn: Advertir sobre los peligros del tabaco

Enforce: Hacer cumplir las prohibiciones sobre publicidad, promoción y patrocinio

Raise: Aumentar los impuestos sobre el tabaco

Las estrategias para el control del tabaco previamente descritas, sin lugar a dudas, son parte de las intervenciones que han demostrado ser costoefectivas y factibles para abatir la epidemia de las ECNT. La completa implementación del CMCT podría evitar cerca de 5.5 millones de muertes en los siguientes 10 años en los países de bajos y medianos ingresos con la mayor carga de la enfermedad por ECNT. ${ }^{38}$

Reconociendo los retos de la aplicación del CMCTOMS en la Región de las Américas, la Organización Panamericana de la Salud (OPS) ha adoptado dos resoluciones: la Resolución CD48.R2 del 30 de septiembre de $2008^{39}$ y la CD50.R6 del 29 de septiembre de $2010^{40}$ las cuales, entre otras recomendaciones, instan a los estados miembros a considerar la ratificación e implementación del CMCT-OMS, dando especial atención al artículo 5.3. del CMCT-OMS y sus directrices, que establecen las medidas para limitar la interacción con la IT y garantizar su transparencia, con el fin de evitar que la misma obstaculice el alcance de las metas de salud pública en el control de tabaco. ${ }^{41}$

\section{La respuesta de México}

En la última década, México ha tenido grandes logros en el control del tabaco. El país firmó el CMCT-OMS el 12 de agosto de 2003, fue aprobado por el Senado de la República el 14 de abril de 2004 y ratificado el 28 de mayo del mismo año, ${ }^{37}$ hechos que llevaron a México a ser el primer país de América en adherirse formalmente a este tratado internacional.

La Secretaría de Salud (SSa), preocupada por el daño en la salud poblacional, los altos costos que el tabaco impone a la sociedad mexicana y como respuesta manifiesta de apoyo al CMCT-OMS, estableció el Plan Nacional de Salud 2007-2012,42 para enfrentar los problemas emergentes mediante la definición explícita de prioridades. Una de ellas es el combate a las adicciones legales e ilegales (tabaco, alcohol y sustancias psicoactivas). El programa de acción específico 2007-2012 sobre prevención y tratamiento de las adicciones en México plantea como objetivo fundamental disminuir el uso, abuso y la dependencia, así como el impacto de las enfermedades y las lesiones que ocasionan en individuos, familias y comunidades, mediante intervenciones de tipo universal, selectivas e indicadas dirigidas a los diversos grupos de población.

En respuesta al Plan de acción de la OMS para la estrategia global para el control de las ECNT 2008-2013, el gobierno federal por acuerdo presidencial crea en 2010, el Consejo Nacional para el Control y Prevención de las ECNT, ${ }^{43}$ el cual tiene como función principal el diseño, implementación y evaluación de programas orientados a abatir la epidemia de ECNT. En 2011, este Consejo presentó el programa "5 Pasos por tu Salud para Vivir Mejor", res de riesgo comunes causantes de las enfermedades crónicas para mejorar la calidad de vida de la población. Utiliza un criterio de inclusión voluntaria que apela a la honestidad y a la pasión de las personas y fomenta la responsabilidad personal y familiar. En el paso 4 de este programa, MIDETE, se recomienda acertadamente evitar el consumo de tabaco. ${ }^{44}$

\footnotetext{
* Programa "5 Pasos por tu Salud para Vivir Mejor" Paso 1: Actívate. (30 minutos de actividad física diariamente en adultos, y 60 minutos en niños y adolescentes). Paso 2: Toma agua (6-8 vasos de agua simple al día). Paso 3: Come frutas y verduras (5 raciones de verduras y frutas al día). Paso 4: Mídete (Mídete física y emocionalmente. Conoce tu peso, talla y estado emocional. Mídete en lo que comes y tomas. No tabaco. No a las drogas. Sexo seguro. Toma tu medicina prescrita. No violencia. Respeta a la mujer. Menos sal. Lactancia materna exclusiva. Usa tu cartilla Nacional de Salud. Afíliate al Seguro Popular). Paso 5: Comparte (comparte con tu familia y tus amigos).
} 
En el plano legislativo se aprobaron e implementaron nuevas leyes federales y locales como la Ley General para el Control del Tabaco (LGCT), ${ }^{45}$ la Ley de Protección a los No Fumadores del Distrito Federal ${ }^{46}$ y el Decreto 078 de la Ley de Salud del Estado de Tabasco. ${ }^{47}$ Se fortalecieron dos áreas rectoras gubernamentales a nivel federal, se creó la Comisión Nacional contra las Adicciones (CONADIC) y se fortaleció el papel del Estado en materia de regulación sanitaria para el control del tabaco a través de la Comisión Federal para la Protección contra Riesgos Sanitarios (COFEPRIS). Desde el punto de vista de prestación de servicios de salud, se amplió la cobertura a través de una red nacional de clínicas de ayuda para dejar de fumar (Centros Nueva Vida) bajo la coordinación del Centro Nacional para la Prevención y el Control de las Adicciones (CENADIC). De manera estratégica se creó la Oficina Nacional para el Control del Tabaco (ONCT) que asesora a los tomadores de decisiones y vincula el trabajo entre el gobierno, las instituciones y la sociedad civil. ${ }^{48}$

México tiene hoy día la oportunidad única para desarrollar, implementar y evaluar una política integral de control del tabaco acorde con las provisiones establecidas en el CMCT-OMS, que se articule con el Plan de acción de la OMS para la estrategia global para el control de las ECNT y de esta manera coadyuve al cumplimiento de los ODM.

\section{Retos y recomendaciones a nivel global}

Sin lugar a dudas, el control del tabaco es una estrategia costo-efectiva para reducir y abatir la epidemia de las enfermedades crónicas y esencial para alcanzar las MDM. ${ }^{49}$ Un estudio de la OMS indica que el precio de implementación de las intervenciones básicas para abatir las ECNT es comparativamente bajo. El costo total para reducir dos factores de riesgo, el consumo de tabaco y el consumo peligroso del alcohol, se estima en 2 billones de dólares anuales para todos los países de bajos y medianos ingresos, lo que significa una inversión de 40 centavos de dólar por persona. ${ }^{2}$

A nivel global, la OMS recomienda incorporar el control del tabaco en la agenda de desarrollo como una estrategia para reducir la pobreza extrema y mejorar la salud de la población mundial. El control del tabaco puede apoyar al cumplimiento de las metas de los ocho objetivos establecidos: ${ }^{49}$

Objetivo 1. Erradicar la pobreza extrema y el hambre. Si los fumadores dejaran de gastar en tabaco mejoraría la economía individual y familiar. Gastarían sus recursos en otros bienes básicos, incluyendo alimentación, educación y salud. Los países afrontarían una menor carga de la enfermedad y podrían reasignar lo que se gasta en la atención de las enfermedades atribuibles al tabaco a un programa dirigido a combatir la pobreza extrema. Adicionalmente, al evitar las muertes prematuras los países mejorarían productividad a nivel nacional.

Objetivo 2. Lograr la enseñanza primaria universal. Si las familias dejan de gastar en tabaco, gastarían sus recursos en educación. Incrementar los niveles de educación en una población es esencial para mejorar su nivel de salud y bienestar. Una población informada es más consciente de los daños a la salud y más receptiva a los programas de prevención y las políticas de salud.

Objetivo 3. Promover la igualdad de género y el empoderamiento de la mujer. Las mujeres juegan un rol importante en las decisiones de salud en el hogar, por lo tanto, mejorar su salud individual implica mejorar la salud de todo su grupo familiar. Adicionalmente, las mujeres representan un alto porcentaje de la fuerza laboral de los países; evitar las muertes prematuras en este grupo poblacional no solo reduciría la carga de enfermedad y muerte sino que mejoraría la productividad nacional.

Objetivos 4 y 5. Reducir la mortalidad infantil y mejorar la salud materna. Evitar el consumo de tabaco en las mujeres reducirá la morbimortalidad perinatal y neonatal, además de evitar las enfermedades de su grupo familiar causadas o agravadas por la exposición a humo de tabaco. Si dejan de gastar en tabaco, podrían asignar el recurso para mejorar su alimentación y la atención en salud.

Objetivo 6. Combatir el VIH / SIDA, el paludismo y otras enfermedades. Evitar el consumo de tabaco y la exposición a su humo evitaría la progresión de enfermedades infecciosas como la TB, las complicaciones que presentan los pacientes con VIH / SIDA y otras enfermedades infecciosas respiratorias.

Objetivo 7. Garantizar la sostenibilidad ambiental. Reducir la deforestación causada por el cultivo de tabaco, la manufactura y los desechos de basura evitarán el impacto negativo en el ambiente y coadyuvará en la sostenibilidad ambiental.

Objetivo 8. Fomentar una alianza mundial para el desarrollo. El Banco Mundial (BM), la Organización para la Cooperación y el Desarrollo Económicos (OCDE) reconocen la carga de la enfermedad causada por el consumo de tabaco y recomiendan el control del tabaco como una estrategia para reducirla. De igual manera, recomiendan una estrategia de cooperación internacional y compromiso de los países para establecer una agenda global para el desarrollo y para la implementación del CMCT-OMS. 
Aún en el peor de los escenarios, donde los recursos económicos son limitados, la mayoría de las recomendaciones son viables y factibles de implementar, si se consideran la voluntad política de los gobiernos, la infraestructura disponible, la capacidad técnica existente, y la participación coordinada de todos los sectores y grupos de interés, incluyendo la sociedad civil organizada y la colectividad en su conjunto.

\section{¿Cómo incorporar el control del tabaco en la agenda de desarrollo de México?}

México requiere considerar y posicionar los objetivos de salud como una prioridad en todas las políticas y niveles de gobierno (federal, estatal y local), con enfoques rectores multisectoriales que involucren, según proceda, a las secretarías de educación, energía, agricultura, deportes, transporte, comunicaciones, urbanismo, medio ambiente, trabajo, industria y comercio, finanzas, y desarrollo social y económico a fin de responder a los factores de riesgo de las ECNT y los factores determinantes básicos de la salud de forma integral y decisiva.

Implementar con decisión, de manera integral, sinérgica y sostenida todas las estrategias MPOWER, como una medida inicial para la ejecución completa del CMCT-OMS. A ocho años de su ratificación, México tiene compromisos pendientes y debe a muy corto plazo:

1. Consolidarse como un país $100 \%$ libre de humo de tabaco, de acuerdo con el artículo 8 del CMCT-OMS y sus directrices.

2. Prohibir totalmente la publicidad, promoción y patrocinio de los productos de tabaco, de acuerdo con el artículo 13 del CMCT-OMS y sus directrices.

3. Incrementar el precio final de los productos de tabaco vía impuestos de acuerdo con lo establecido en el artículo 6 del CMCT-OMS y sus directrices.

4. Asegurar la sanción efectiva de aquellos que comercian de manera ilícita los productos de tabaco y los venden a los menores de edad y al menudeo (artículos 15 y 16 del CMCT-OMS y sus directrices, respectivamente).

5. Implementar todas las directrices del artículo 11 del CMCT-OMS en torno al empaquetado y etiquetado de productos de tabaco.

Vincular el sector gubernamental con un grupo de trabajo intersectorial de nivel técnico, académico y de investigación que:

1. En primer lugar fortalezcan los sistemas de información, monitoreo y vigilancia de la epidemia de ECNT y sus factores de riesgo, creando un Observa- torio Mexicano que integre tanto factores de riesgo como enfermedades.

2. Evalúen el impacto de las estrategias globales y locales implementadas desde una perspectiva ampliada (epidemiológica, económica, ambiental y social entre otras).

3. Impulsen programas académicos para la formación y capacitación de recursos humanos de tipo multidisciplinario con posibilidad de incorporarse en los programas preventivos y de atención médica a nivel local, nacional o regional; $\mathrm{y}$

4. Fomenten grupos de investigación interinstitucional con capacidad de vinculación e intercambio a nivel nacional e internacional.

Gestionar nuevas fuentes de financiamiento, recursos económicos e infraestructura a través de cooperaciones internacionales o nacionales que permitan operar los programas preventivos y las estrategias de acción sin apartarse de los parámetros de ética, transparencia y rendición de cuentas. Abogar por el etiquetado de los recursos económicos procedentes de la recaudación de impuestos para el tratamiento de la adicción y las enfermedades atribuibles al tabaco que en la actualidad no están cubiertos por la seguridad social ni el Sistema de Protección Social en Salud (SPSS). Lograr una mejor negociación en la asignación de recursos económicos para todos los sectores involucrados en alcanzar los objetivos de salud.

Asegurar el acceso a los servicios de salud y la cobertura universal de los pacientes con adicción al tabaco o con padecimientos relacionados con el consumo o exposición a su humo, buscando la incorporación del tratamiento farmacológico de la adicción al cuadro básico de medicamentos y la implementación de estrategias de diagnóstico temprano y tamizaje de las enfermedades, específicamente para el cáncer.

Incorporar las bases y conceptos de la mercadotecnia social en los proyectos del sector salud que generen un verdadero cambio de comportamiento de la sociedad mexicana, especialmente en los grupos vulnerables ya identificados, creando un colectivo de salud y bienestar. Estas estrategias deben ir más allá de una campaña asilada en los medios masivos de comunicación, deben ser innovadoras y progresistas de acuerdo con el desarrollo tecnológico e incluir estrategias de investigación de mercados, monitoreo y evaluación.

Todo este esfuerzo no será posible si no se es equitativo e incluyente y se incorpora a la sociedad mexicana en su conjunto. Vincular y trabajar de manera armónica con la sociedad civil organizada en la búsqueda de proyectos e iniciativas para la resolución de necesidades sentidas a corto plazo e incorporar acciones dirigidas a 
grupos vulnerables con participación activa de las comunidades tanto en la ejecución como en la evaluación de programas.

Involucrar de manera activa a la población civil en la denuncia de violaciones a la legislación y normatividad. Una respuesta oportuna y efectiva de las instituciones a la denuncia ciudadana confidencial y anónima es fundamental para incentivar la utilización de las líneas existentes y garantizar la correcta aplicación de la normatividad federal y local.

Estas son sólo algunas de las estrategias orientadas a fortalecer la capacidad de la sociedad mexicana para decidir por alternativas más sanas y adoptar modos de vida que propicien bienestar y buen estado de salud.

Un poco más de una década nos separa del inicio del siglo XXI. La historia nos demuestra que las epidemias son cambiantes en todas sus dimensiones, por lo tanto, el quehacer de la salud pública, nuestro quehacer diario, no puede detenerse en el tiempo, romper viejos paradigmas y conceptualizar los problemas de salud. Al considerar la presencia simultánea de factores y circunstancias de orden global como las crisis económicas, los desastres naturales generados por el cambio climático, los nuevos sistemas de gobierno, la escasez de recursos económicos, el desempleo, la discriminación, así como los factores locales que afectan de manera particular a nuestros países serán los retos que debemos superar para disminuir la prevalencia global de consumo de tabaco al 5\% y alcanzar la meta propuesta para el 2040, un mundo esencialmente libre de tabaco.

Declaración de conflicto de intereses: La autora declaró no tener conflicto de intereses.

\section{Referencias}

I. World Health Organization. Global status report on Noncommunicable Diseases. Geneva:WHO, 2011

2. World Economic Forum, Harvard School of Public Health. The Global Economic Burden of Non Communicable Diseases. Geneva: World Economic Forum, 20II.

3. World Health Organization. WHO report on the global tobacco epidemic, 2008: the MPOWER package. Geneva, World Health Organization. ISBN: 978924 I59628 2. [Consultado 20II septiembre 2I]. Disponible en: http://www.who.int/tobacco/mpower/gtcr_download/ en/index.html.

4. World Health Organization. WHO report on the global tobacco epidemic, 20I I: warning about the dangers of tobacco. [Consultado 2012 febrero 5]. Disponible en: http://www.who.int/tobacco/global_ report/20I I/en/index.html

5. World Health Organization. International consultation on environmental tobacco smoke (ETS) and child health. 1999. [Consultado 20I I septiembre 2I]. Disponible en: http://www.who.int/tobacco/ resources/publications/ets/en/

6. US Department of Health and Human Services. The health consequences of involuntary exposure to Tobacco Smoke: A Report of the Surgeon General. Atlanta (GA): US Department of Health and Human Services. Centers for Disease Control and Prevention. National Center for Chronic Disease Prevention and Health Promotion, Office on Smoking and Health, 2006.

7. World Bank. 1999. Curbing the epidemic: governments and the economics of tobacco control. The International Bank for Reconstruction and Development/THE WORLD BANK, $1818 \mathrm{H}$ Street, N.W.

Washington, D.C. 20433. [Consultado 20II septiembre 2I]. Disponible en: http://www.usaid.gov/policy/ads/200/tobacco.pdf

8. Jha P. Avoidable global cancer deaths and total deaths from smoking. Nat Rev Cancer 2009;9 (9):655-664.

9. Mathers CD, Loncar D. Projections of global mortality and burden of disease from 2002 to 2030. PLoS Medicine 2006; 3 (I I):e 442.

10. Van Zyl-Smit, Bwnet L, Pai M, Yew WW. The Convergence of the Global Smoking, COPD, Tuberculosis, HIV, and Respiratory Infections Epidemics. Infect Dis Clin N Am 2010; 24: 693-703.

II. Van Zyl-Smit RN, Pai M, Yew WW, Leung CC, Zumla A, Bateman ED, Dneda K. Global lung Health: the colliding epidemics of tuberculosis, tobacco smoking, HIV, and COPD. Eur Respir J 20I0; 35: 27-33.

12. Farr BM, BarLETT cl, Wadsworth J, Miller DL. Risk factors for community-adquired pneumonia diagnosed upon hospital admission. British Thoracic Society Pneumonia Study Group. Resp Med 2000;94(10):954-963. I3. Doll R, Peto R, Boreman J, Sutherland I. Mortality in relation to smoking: 50 years' observations on male British doctors. $\mathrm{Br}$ J Cancer 2005; 92:426-429.

14. US Department of Health and Human Services (USDHHS). The health consequences of smoking: A Report of the Surgeon General. Atlanta (GA): US Department of Health and Human Services. Centers for Disease Control and Prevention. National Center for Chronic Disease Prevention and Health Promotion, Office on Smoking and Health, 2004. 15. Lopez AD, Collishaw NE, Piha T. A Descriptive model of the cigarette epidemic in developed countries. Tobacco Control 1994; 3(3): 242-247. 16. US Department of Health and Human Services (USDHHS). The Health Benefits of Smoking Cessation A Report of the Surgeon General, 1990. US Department of Health and Human Services. Centers for Disease Control and Prevention. National Center for Chronic Disease Prevention and Health Promotion, Office on Smoking and Health, 1990. (DHHS publication No (CDC) 90-8416).

17. ACS, WLF, Shafey O, Eriksen M, Ross H, Mackay J. The Tobacco Atlas. Third Edition, 2009. [Consultado 20II septiembre 2I]. Disponible en: http://www.tobaccoatlas.org/

18. World Health Organization. 20II. Systematic review of the link between tobacco and Poverty, Geneva: WHO, 20II

19. Jha P, Peto R, Zatonski W, Boreham J, Jarvis MJ, Lopez AD. Social inequalities in male mortality, and in male mortality from smoking: indirect estimation from national death rates in England and Wales, Poland, and North America. Lancet 2006;368(9533):367-370. 20. World Health Organization. Convenio Marco de la OMS para el Control del Tabaco. Geneva 2003. [Consultado 20II septiembre 2I]. Disponible en: http://www.who.int/tobacco/framework/en/

21. World Health Organization. Tobacco industry interference with tobacco control. I.Tobacco industry - legislation. 2. Tobacco industry - trends. 3.Smoking - prevention and control. 4.Smoking - economics. 5.Lobbying. 6.Tobacco - supply and distribution. 7.Policy making. I.World Health Organization. II.WHO Tobacco Free Initiative. III. Conference of the Parties to the WHO Framework Convention on Tobacco Control. 22. Organización Panamericana de la Salud; Instituto Nacional de Salud Pública (MX). Encuesta Global de Tabaquismo en Adultos. México 2009. Cuernavaca (México): INSP/OPS, 2010.

23. Reynales-Shigematsu LM, Valdés-Salgado R, Rodríguez-Bolaños R, Lazcano- Ponce E, Hernández-Ávila M. Encuesta de Tabaquismo en Jóvenes en México. Análisis descriptivo 2003, 2005, 2006, 2008. Cuernavaca, México: Instituto Nacional de Salud Pública, 2009. 
24. Secretaría de Salud. Encuesta Nacional de Adicciones 2008. México: SSA, 2009.

25. Barrientos GT, Reynales SLM, Ávila TE et al. Exposición al humo de tabaco en hogares de la Ciudad de México: análisis de nicotina ambiental y en cabello de niños y mujeres. Salud Pública Mex 2007 b;49 (supl 2):S224- S232.

26. Reynales-Shigematsu LM, Vázquez JH, Lazcano E. Encuesta Mundial de Tabaquismo en Estudiantes de la Salud, México 2006. Salud Publica Mex 2007; 49 supl. 2:194-204.

27. Olaíz G, Rivera J, Shamah T, Rojas R, Villalpando S, Hernández M, et al. Encuesta Nacional de Salud 2006. México: Instituto Nacional de Salud Pública, 2006.

28. U.S. Department of Health and Human Services. How Tobacco Smoke Causes Disease: The Biology and Behavioral Basis for SmokingAttributable Disease: A Report of the Surgeon General. Atlanta, GA: U.S. Department of Health and Human Services, Centers for Disease Control and Prevention, National Center for Chronic Disease Prevention and Health Promotion, Office on Smoking and Health, 2010.

29. Valdés R. Las cifras de la epidemia. Daños a la salud y mortalidad atribuible. En: Primer informe sobre combate al tabaquismo. México ante el convenio Marco para el Control del Tabaco. México. Cuernavaca: Instituto Nacional de Salud Pública, 2005:29-4I.

30. SSA. SINAIS Sistema Nacional de Información en Salud, Tabla Dinámica de Defunciones, 1979-2009. [Consultado 201I septiembre 2I]. Disponible en: http://www.sinais.salud.gob.mx/basesdedatos/index. html\#dinamica

31. Sáenz de Miera-Juárez B, 15ht World Conference Tobacco or Health; 2012 March 20-24; Singapure, Singapure. Las repercusiones del consumo de tabaco en la distribución del gasto de los hogares mexicanos. Documento interno de trabajo.

32. Análisis de la Encuesta Nacional de Ingresos y Gastos de los Hogares (ENIGH) 2010 (INEGI). [Consultado 20II septiembre 2I]. Disponible en www.inegi.gob.mx).

33. Reynales-Shigematsu LM. Costos de atención médica atribuibles al consumo de tabaco en México. ISPOR 3rd Latin America Conference. Building Networks across the institutions for access to Health care in Latin America; 20II september 8-10; Mexico City, Mexico.

34. Naciones Unidas. Declaración Política de la Reunión de Alto Nivel de la Asamblea General sobre la Prevención y el Control de las Enfermedades No Transmisibles. Septiembre de 20I I. Ginebra: UN, 20II 35. Mamudu HM. UN resolution on the prevention and control of noncommunicable diseases: an opportunity for global action. Glob Public Health 20I I;6 (4):347-353.

36. Naciones Unidas. Objetivos de Desarrollo del Milenio. Informe 201 I. [Consultado 2012 enero 5] Disponible en: http://www.un.org/spanish/ millenniumgoals/pdf/MDG_Report_20II_SP

37. Partes en el Convenio Marco de la OMS para el control del Tabaco. [Consultado 2012 enero 5] Disponible en: http://www.who.int/fctc/ signatories_parties/es/index.html

38. Beaglehole R, Bonita R, Horton R, Adams C, Alleyne G, Asaria P, et al. Priority actions for the non-communicable disease crisis. Lancet 201 I; 377(9775): I438-1447.
39. Organización Panamericana de la Salud 48.o Consejo Directivo. Resolución CD48.R2 - El Convenio Marco de la OMS para el control del tabaco: oportunidades y retos para su implementación en la Región de las Américas. [Consultado 2012 febrero I2] Disponible en: http:// www.paho.org/spanish/gov/cd/cd48.r2-s.pdf

40. Organización Panamericana de la Salud 50.o Consejo Directivo. Resolución CD50.R6 - Fortalecimiento de la capacidad de los Estados Miembros para Implementar las disposiciones y directrices del Convenio Marco de la OMS para el control del tabaco. [Consultado 2012 febrero 12] Disponible en: http://new.paho.org/hq/dmdocuments/2010/CD50. R6-s.pdf

4I. Organización Panamericana de la Salud. Informe sobre Control del Tabaco 20II. México. [Consultado 2012 febrero 5]. Disponible en: http:// new.paho.org/hg/index.php?option=com content\&task=view\&id=4457\&it emid $=123 \mid$ \&lang $=$ es.

42. Secretaría de Salud. Programa Nacional de Salud 2007-20I2. Por un México sano: construyendo alianzas para una mejor salud. Secretaría de Salud. México, D.F. [Consultado 20II diciembre I2] Disponible en: http:// alianza.salud.gob.mx/descargas/pdf/pns_version_completa.pdf 43. Versión estenográfica de la Sesión de Instalación del Consejo Nacional para la Prevención y Control de las Enfermedades Crónicas no Transmisibles, presidida esta mañana por el doctor José Ángel Córdova Villalobos, Secretario de Salud Federal, en el Salón del Consejo Nacional de Salud "Guillermo Soberón Acevedo", de esta Secretaría [Consultado 20II Diciembre 12] Disponible en: http://www.salud.gob.mx/unidades/ dgcs/sala_noticias/discursos/2010_05_I2-prevencion.htm

44. Barriguete A. 5 Pasos por tu Salud para Vivir Mejor 2009 - 2012. Presentación disponible en: [Consultado 20II diciembre 12] Disponible en: http://www.promocion.salud.gob.mx/red/descargables/Disco_gto_ web_con_imagina/dia_10/conferencias/5\%20Pasos\%20por\%20Tu\%20 Salud\%20MUNICIPIO\%20Barriguete[I].pdf 45. Ley General para el Control del Tabaco. Diario Oficial de la Federación. 30/04/2008. México, DF: Congreso General, 2008. 46. Ley de Protección a los no fumadores del Distrito Federal. [Consultado 20II diciembre 12] Disponible en: http://www.conadic.salud. gob.mx/pdfs/onct/LeyProteccionSalud_NoFumadoresDF.pdf 47. Ley de Salud del Estado de Tabasco. [Consultado 20II Diciembre 12] Disponible en: http://www.tabasco.gob.mx/noticias/vernotas_ sp.php?id=4662

48. Secretaría de Salud. Informe de Actividades 20I I: Avances en la Prevención y Atención de las Adicciones. Acciones Estratégicas, Evaluación y seguimiento de los Programas Nacionales contra las Adicciones.México: Secetaría de Salud, Comisión Nacional contra las Adicciones, 20II.

49. World Health Organization. The Millennium development goals and tobacco control: an opportunity for global partnership / Katharine M. Esson, Stephen R. Leeder. I. Development 2. Goals 3. Tobacco adverse effects 4. Smoking - prevention and control 5. Tobacco industry - legislation 6. Socioeconomic factors 7. International cooperation I. Leeder, Stephen R. II. Title. (NLM classification: WA 530.I). 